\title{
THE ALBERT QUADRATIC FORM FOR AN ALGEBRA OF DEGREE FOUR
}

\author{
P. MAMMONE AND D. B. SHAPIRO \\ (Communicated by Donald S. Passman)
}

\begin{abstract}
Suppose $K$ is a field and the $K$-algebra $A$ is expressed as a tensor product of two quaternion algebras $A \cong H_{1} \otimes H_{2}$. Let $N_{i}$ be the norm form on $H_{i}$ and define the "Albert form" $\alpha_{A}$ to. be the 6-dimensional quadratic form determined by $\alpha_{A} \perp\langle 1,-1\rangle \cong N_{1} \perp-N_{2}$. In [Adv. in Math. 48 (1983), 149-165] Jacobson proved: (1) any two Albert forms for $A$ are similar; (2) if $A$ and $B$ are algebras of this type, then $A \cong B$ if and only if $\alpha_{A}$ and $\alpha_{B}$ are similar.

The authors prove this result using quadratic forms and Clifford algebras, avoiding the application of Jacobson's theory of Jordan norms.
\end{abstract}

\section{INTRODUCTION}

Suppose $A$ is a central simple (associative) algebra of degree 4 and exponent 2 over a field $K$. By a well-known theorem of Albert (see [A1, p. 174 or R]) such an algebra is expressible as a tensor product of two quaternion algebras. Following the idea of Jacobson [J] we define the "Albert quadratic form" $\alpha_{A}$. First choose an expression $A \cong H_{1} \otimes H_{2}$, where $H_{1}$ and $H_{2}$ are quaternion algebras, and let $N_{i}$ be the usual norm form on $H_{i}$, defined by $N_{i}(x)=x \bar{x}$. Then the form $N_{1} \perp-N_{2}$ is isotropic, so it contains a copy of the hyperbolic plane $H$ as a subform. Define $\alpha_{A}$ to be the 6-dimensional quadratic form satisfying $\alpha_{A} \perp \mathbf{H} \approx N_{1} \perp-N_{2}$. Our definition differs from Jacobson's in the case $K$ has characteristic 2, correcting an error in [J] (see $\S 3$ below).

The main result is that the form $\alpha_{A}$ is well defined up to similarity, independent of the decomposition of $A$. Two quadratic forms $\alpha, \beta$ over $K$ are said to be similar if $\alpha$ is isometric to a scalar multiple of $\beta$, that is, if $\alpha \approx\langle x\rangle \beta$ for some $x \in K^{*}$.

Jacobson's Theorem [J, THEOREM 3.12]. Let $A, B$ be central simple algebras of degree 4 and exponent 2 over a field $K$.

(i) Any two Albert forms for $A$ are similar.

(ii) $A \cong B$ if and only if $\alpha_{A}$ and $\alpha_{B}$ are similar.

Received by the editors March 24, 1988.

1980 Mathematics Subject Classification (1985 Revision). Primary 10C05; Secondary 16A46. 
(iii) The index of $A$ is 4, 2 or 1 according as the Witt index of $\alpha_{A}$ is 0,1 or 3.

Here the (Schur) index of $A$ is the degree of the division algebra part of $A$. Jacobson derives this result from his theory of Jordan norms applied to the Jordan algebra of symmetric elements in $A$. In this note we give a different proof of the theorem using quadratic forms and Clifford algebras. We first prove the theorem in the case char $K \neq 2$ using techniques of quadratic form theory as in [L and $\mathrm{S}$ ]. When char $K=2$ we prove the theorem by establishing the analogs of each of the earlier steps.

This work forms part of the first author's doctoral dissertation [M1]. It is a pleasure to thank $\mathrm{J}$. P. Tignol for many stimulating conversations and $\mathrm{A}$. Wadsworth for several valuable comments.

\section{Characteristic not two}

Suppose $K$ is a field with char $K \neq 2$. We follow the notations for quadratic forms and Witt rings found in [L and S]. If $\varphi$ is a (nonsingular) quadratic form of dimension $n$ of $K$, the discriminant is $d \varphi=(-1)^{n(n-1) / 2} \operatorname{det} \varphi$ in $K^{*} / K^{* 2}$. The discriminant induces a map on the Witt ring $d: W K \rightarrow K^{*} / K^{* 2}$.

The Witt invariant $c(\varphi)$ is defined to be the class in the Brauer group $\operatorname{Br}(K)$ of a certain Clifford algebra. Rules for computing $c(\varphi)$ are given in [L, p. 121 and $\mathrm{S}, \mathrm{p}$. 81]. The Witt invariant induces a map $c: W K \rightarrow \operatorname{Br}(K)$. For example for $a, b \in K^{*}$ let $(a, b)$ denote the class of the quaternion algebra $(a, b) / K$ in $\operatorname{Br}(K)$. The norm form of this algebra is the 2-fold Pfister form $\langle\langle-a,-b\rangle\rangle=\langle 1,-a,-b, a b\rangle$, and we have $c(\langle\langle-a,-b\rangle\rangle)=(a, b)$. We follow the usual abuse of notation writing $(a, b)$ both for the quaternion algebra and for its class in $\operatorname{Br}(K)$, and writing $\varphi$ both for the quadratic form and for its class in $W(K)$.

The ideal $I K$ of even dimensional forms in $W K$ and its powers $I^{n} K$ play a fundamental role in this theory. For example $\varphi \in I^{2} K$ iff $\varphi \in I K$ and $d \varphi=\langle 1\rangle$. It is also easy to check that if $\varphi \in I^{3} K$ then $\varphi \in I^{2} K$ and $c(\varphi)=1$. Pfister [P] proved a partial converse. We state the parts of Pfister's theorem which we will use later.

2.1 Proposition (Pfister). (i) If $\varphi \in I^{2} K, c(\varphi)=1$ and if $\operatorname{dim} \varphi \leq 12$ then $\varphi \in I^{3} K$.

(ii) If $\operatorname{dim} \varphi=6, d \varphi=\langle 1\rangle$ and $c(\varphi)$ is quaternion then $\varphi$ is isotropic.

(iii) If $\operatorname{dim} \varphi=10, d \varphi=\langle 1\rangle$ and $c(\varphi)=1$ then $\varphi$ is isotropic.

Proofs of Pfister's theorem are given in [P, Satz 14 and S, pp. 90-91]. Merkurjev has proved the general result, without restrictions on dimension: if $\varphi \in I^{2} K$ and $c(\varphi)=1$ then $\varphi \in I^{3} K$. We will not use that difficult theorem here. 
2.2 Lemma (WADSWORTH). Let $\varphi$ and $\psi$ be quadratic forms over $K$ and suppose $\operatorname{dim} \varphi=\operatorname{dim} \psi=4$ and $d \varphi=d \psi=\langle d\rangle$. Let $L=K(\sqrt{d})$. If $\varphi \otimes L$ and $\psi \otimes L$ are similar over $L$ then $\varphi$ and $\psi$ are similar over $K$.

The proof appears in [W1, Theorem 7].

2.3 Proposition. Suppose $\varphi$ and $\psi$ and quadratic forms over $K$ with $\operatorname{dim} \varphi=$ $\operatorname{dim} \psi=4, d \varphi=d \psi$ and $c(\varphi)=c(\psi)$. Then $\varphi$ and $\psi$ are similar.

Proof. Suppose $d \varphi=d \psi=\langle d\rangle$. If $\langle d\rangle=\langle 1\rangle$ the result is easy to prove, as in [S, p. 88, Theorem 14.1]. If $\langle d\rangle \neq\langle 1\rangle$ we can extend scalars to the field $L=K(\sqrt{d})$, use the first case to conclude that $\varphi \otimes L$ and $\psi \otimes L$ are similar over $L$ and apply the previous lemmà.

2.4 Proposition. Suppose $\alpha$ and $\beta$ are quadratic forms over $K$ with $\operatorname{dim} \alpha=$ $\operatorname{dim} \beta=6, d \alpha=d \beta=\langle 1\rangle$ and $c(\alpha)=c(\beta)$. Then $\alpha$ and $\beta$ are similar.

Proof. By Proposition 2.1(i) applied to $\alpha \perp-\beta$ we see that the hypothesis implies $\alpha \equiv \beta\left(\bmod I^{3} K\right)$. We may scale $\alpha$ and $\beta$ independently, so let us assume they represent 1 . Say $\alpha \approx\langle 1\rangle \perp \alpha_{1}$ and $\beta \approx\langle 1\rangle \perp \beta_{1}$. Then $\alpha_{1} \equiv$ $\beta_{1}\left(\bmod I^{3} K\right)$ so that $\alpha_{1} \perp-\beta_{1} \in I^{3} K$ is 10 -dimensional. By Proposition 2.1(iii) this form must be isotropic. Then $\alpha_{1}$ and $\beta_{1}$ represent a common value, say $\alpha_{1} \approx\langle d\rangle \perp \alpha_{2}$ and $\beta_{1} \approx\langle d\rangle \perp \beta_{2}$. Then $\alpha_{2} \equiv \beta_{2}\left(\bmod I^{3} K\right)$ and $\operatorname{dim} \alpha_{2}=\operatorname{dim} \beta_{2}=4$. We conclude from Proposition 2.3 that these forms are similar. Say $\alpha_{2} \approx\langle x\rangle \beta_{2}$ for some $x \in K^{*}$. Then $c\left(\alpha_{2}\right)=c\left(\langle x\rangle \beta_{2}\right)=$ $c\left(\beta_{2}\right)\left(x, d \beta_{2}\right)$. Since $c\left(\alpha_{2}\right)=c\left(\beta_{2}\right)$ and $d \beta_{2}=\langle-d\rangle$ we have $1=(x$, $-d)$. Then the form form $\langle\langle-x, d\rangle\rangle$ is hyperbolic so that $\langle x\rangle\langle 1, d\rangle \approx\langle 1, d\rangle$. Therefore $\langle x\rangle \beta \approx\langle x\rangle\left(\langle 1, d\rangle \perp \beta_{2}\right) \approx\langle 1, d\rangle \perp \alpha_{2} \approx \alpha$.

Now we can prove Jacobson's Theorem. Suppose $A \cong\left(a_{1}, b_{1}\right) \otimes\left(a_{2}, b_{2}\right)$ is the tensor product of two quaternion algebras over $K$. By definition the Albert form $\alpha$ is determined by: $\mathbf{H} \perp \alpha \approx N_{1} \perp-N_{2}$, where $N_{i} \approx\left\langle\left\langle-a_{i}, b_{i}\right\rangle\right\rangle$ is the norm form of the quaternion algebra. Therefore $\alpha \approx\left\langle-a_{1},-b_{1}, a_{1} b_{1}, a_{2}, b_{2}\right.$, $\left.-a_{2} b_{2}\right\rangle$. Then $\operatorname{dim} \alpha=6, d \alpha=\langle 1\rangle$ and $c(\alpha)=c\left(N_{1} \perp-N_{2}\right)=c\left(N_{1}\right) c\left(N_{2}\right)=$ $\left(a_{1}, b_{1}\right)\left(a_{2}, b_{2}\right)=[A]$.

Suppose $B$ is another algebra decomposed as a tensor product of quaternions, and let $\beta$ be the corresponding Albert form. If $A \cong B$ then $c(\alpha)=$ $[A]=[B]=c(\beta)$ and Proposition 2.4 implies $\alpha$ and $\beta$ are similar. Conversely, if $\alpha$ and $\beta$ are similar, say $\alpha \approx\langle x\rangle \beta$, then $[A]=c(\alpha)=c(\langle x\rangle \beta)=$ $c(\beta)(x, d \beta)=c(\beta)=[B]$, and we have $A \cong B$. This proves parts (i) and (ii) of Jacobson's Theorem.

The nontrivial part of (iii) is to prove that if the Albert form $\alpha_{A}$ is anisotropic then $A$ is a division algebra. In addition to his own proof, Jacobson refers to proofs by Albert, Tamagawa and Seligman. The result was also proved by Pfister [P]. In fact it is equivalent to Proposition 2.1(ii) stated above. This completes our proof in the case $\operatorname{char} K \neq 2$. 


\section{Characteristic two}

Let $K$ be a field of characteristic two. We follow most of the notations for quadratic forms, Witt groups and quaternion algebras found in [B]. If the quadratic form $\varphi=\perp_{i=1}^{n}\left\langle c_{i}\right\rangle\left[a_{i}, b_{i}\right]$, the Arf invariant of $\varphi$ is $\Delta(\varphi)=\sum_{i=1}^{n} a_{i} b_{i}$ in $K / \wp(K)$ and the Witt invariant $c(\varphi)$ is the class in $\operatorname{Br}(K)$ of the tensor product of quaternion algebras $\bigotimes_{i=1}^{n}\left(b_{i}, a_{i} b_{i}\right]$. In particular if $\varphi=\langle\langle a, b]]$ is the norm form of the quaternion algebra $H=(a, b]$, then $c(\varphi)=[H]$. These invariants induce maps on the Witt group $W q(K)$. We follow the usual abuse of notation writing $(b, a]$ both for the quaternion algebra and for its class in $\operatorname{Br}(K)$, and writing $\varphi$ both for the quadratic form and its class in $W q(K)$.

3.1 Lemma. The sequences

$$
\begin{aligned}
& 0 \rightarrow I W q(K) \rightarrow W q(K) \stackrel{\Delta}{\rightarrow} K / \wp(K) \\
& 0 \rightarrow I^{2} W q(K) \rightarrow I W q(K) \stackrel{c}{\rightarrow} \operatorname{Br}(K)
\end{aligned}
$$

are exact.

Proof. See [Sa, Theorem 2, p. 152].

The next Lemma is the characteristic 2 analogue of Wadsworth's Lemma 2.2.

3.2 Lemma. Let $\varphi$ and $\psi$ be quadratic forms over $K$ and suppose $\operatorname{dim} \varphi=$ $\operatorname{dim} \psi=4$ and $\Delta(\varphi)=\Delta(\psi)=\Delta$. Let $L=K\left(\wp^{-1}(\Delta)\right)$. If $\varphi \otimes L$ is similar to $\psi \otimes L$ over $L$ then $\varphi$ and $\psi$ are similar over $K$.

Proof. See [M1, p. 79 or M2].

3.3 Proposition. Suppose $\varphi$ and $\psi$ are quadratic forms over $K$ with $\operatorname{dim} \varphi=$ $\operatorname{dim} \psi=4$ and $\varphi \equiv \psi\left(\bmod I^{2} W q(K)\right)$. Then $\varphi$ and $\psi$ are similar.

Proof. The congruence condition and Lemma 3.1 imply that $\Delta(\varphi)=\Delta(\psi)$ and $c(\varphi)=c(\psi)$. Let $L=K\left(\wp^{-1}(\Delta)\right)$ where $\Delta=\Delta(\varphi)$. Since the Arf invariants become 0 over $L$ we can express $\varphi \otimes L \approx\left\langle c_{1}\right\rangle\left\langle\left\langle b_{1}, a_{1}\right]\right]$ and $\psi \otimes L \approx\left\langle c_{1}\right\rangle\left\langle\left\langle b_{2}, a_{2}\right]\right]$, for some $a_{i}, b_{i}, c_{i} \in L^{*}$. Computing the Witt invariants over $L$ we find that $\left(b_{1}, a_{1}\right]=c(\varphi \otimes L)=c(\psi \otimes L)=\left(b_{2}, a_{2}\right]$ in $\operatorname{Br}(L)$. Then those quaternion algebras are isomorphic so their norm forms are isometric: $\left\langle\left\langle b_{1}, a_{1}\right]\right] \approx\left\langle\left\langle b_{2}, a_{2}\right]\right]$ over $L$. Therefore $\varphi \otimes L \approx\left\langle c_{1} c_{2}\right\rangle \psi \otimes L$, and Lemma 3.2 completes the proof.

3.4 Lemma. Suppose $\operatorname{dim} \varphi=6, \Delta(\varphi)=0$. Then $c(\varphi)$ is quaternion iff $\varphi$ is isotropic.

Proof. This is part of the characteristic 2 analog of Pfister's Theorem 2.1 above, proved by Baeza. See [B, Theorem 4.18, p. 129].

3.5 Proposition. Suppose $\alpha$ and $\beta$ are quadratic forms over $K$ with $\operatorname{dim} \alpha=$ $\operatorname{dim} \beta=6, \Delta(\alpha)=\Delta(\beta)=0$ and $c(\alpha)=c(\beta)$. Then $\alpha$ and $\beta$ are similar. 
Proof. By Lemma 3.1 applied to $\alpha \perp-\beta$ we see that the hypothesis implies $\alpha \equiv \beta\left(\bmod I^{2} W q(K)\right)$. We may scale $\alpha$ to assume $\alpha=[1, a] \perp \alpha_{1}$. Let $L=$ $K\left(\wp^{-1}(a)\right)$ so that $\alpha \otimes L$ is isotropic. By Lemma 3.4 applied twice we find that $\beta \otimes L$ is isotropic too. By [B, Theorem 4.2, p. 121] we know that $\beta$ contains a subform isometric to $\langle y\rangle[1, a]$, for some $y \in K^{*}$. Then $\langle y\rangle \beta \approx[1, a] \perp \beta_{1}$ for some form $\beta_{1}$. Then $\alpha \equiv \beta \equiv\langle y\rangle \beta\left(\bmod I^{2} W q(K)\right)$ implies that $\alpha_{1} \equiv \beta_{1}$ $\left(\bmod I^{2} W q(K)\right)$, and Proposition 3.3 shows that $\alpha_{1}$ and $\beta_{1}$ are similar. Say $\alpha_{1} \approx\langle x\rangle \beta_{1}$ for some $x \in K^{*}$. Then $c\left(\alpha_{1}\right)=c\left(\langle x\rangle \beta_{1}\right)=c\left(\beta_{1}\right)\left(x, \Delta\left(\beta_{1}\right)\right]$. Since $c\left(\alpha_{1}\right)=c\left(\beta_{1}\right)$ and $\Delta\left(\beta_{1}\right)=a$ we have $1=(x, a]$. This implies that the form $[1, a]$ represents $x$, so that $\langle x\rangle[1, a] \approx[1, a]$. Therefore $\langle x y\rangle \beta \approx$ $\langle x\rangle\left([1, a] \perp \beta_{1}\right) \approx[1, a] \perp \alpha_{1} \approx \alpha$.

Now we can prove Jacobson's Theorem. Suppose $A \cong\left(b_{1}, a_{1}\right] \otimes\left(b_{2}, a_{2}\right]$ is the tensor product of two quaternion algebras over $K$. The Albert quadratic form $\alpha$ is defined from the equation $\mathbf{H} \perp \alpha \approx N_{1} \perp-N_{2}$ were $N_{1} \approx\left\langle\left\langle b_{i}, a_{i}\right]\right]$ is the norm of the quaternion algebra. Cancelling $\mathbf{H}$ shows that $\alpha \approx\left[1, a_{1}+\right.$ $\left.a_{2}\right] \perp\left\langle b_{1}\right\rangle\left[1, a_{1}\right] \perp\left\langle b_{2}\right\rangle\left[1, a_{2}\right]$. Then $\operatorname{dim} \alpha=6, \Delta(\alpha)=0$ and $c(\alpha)=c\left(N_{1} \perp-\right.$ $\left.N_{2}\right)=c\left(N_{1}\right) c\left(N_{2}\right)=\left(b_{1}, a_{1}\right]\left(b_{2}, a_{2}\right]=[A]$. Note that there is an error in [J] concerning the calculation of $\alpha$. On p. 155 line 18 when $\operatorname{char} \Phi=2$ the space $\mathscr{L}$ has dimension 5 , not 6 .

The proof of parts (i) and (ii) of Jacobson's Theorem is nearly identical with the case done in $\S 2$ above. Part (iii) is essentially done in Baeza's Lemma 3.4 above, as well as in the reference mentioned in [J].

Final remark. A. Wadsworth has informed us that the characteristic 2 case of Jacobson's Theorem can be deduced directly from the characteristic 0 case using a valuation argument in the style of his work in [W2]. Here is a brief outline of these ideas.

Let $F$ be a Henselian valued field with valuation $v$, valuation ring $V$, residue field $\bar{F}$ and value group $\Gamma$. If $q^{\prime}$ is a nondegenerate quadratic form over $\bar{F}$, let $q$ be a "lift" of $q^{\prime}$ considered as a quadratic form over $F$. Any two such lifts are isometric by [K, Satz 3.3].

1. Lemma. (1) If $q^{\prime}$ is anisotropic then $q$ is anisotropic and for any $c \in D_{F}(q)$ we have $v(c) \in 2 \Gamma$.

(2) If $q_{1}^{\prime}$ and $q_{2}^{\prime}$ are similar nondegenerate quadratic forms over $\bar{F}$, then their lifts $q_{1}$ and $q_{2}$ are similar over $F$.

Proof. (1) uses a standard valuation argument, and (2) follows from (1).

2. Proposition. Let $D^{\prime}$ be a finite dimensional $\bar{F}$-central division algebra. Then $D^{\prime}$ has a unique "inertial lift" $D$ which is an $F$-central division algebra satisfying $[D: F]=\left[D^{\prime}: \bar{F}\right]$ and (using the unique extension of $v$ to $D$ ), $\bar{D} \cong D^{\prime}$.

Proof. See [JW, §2].

Now we can deduce Jacobson's Theorem in characteristic 2. Suppose $A^{\prime}$ and $B^{\prime}$ are $K$-algebras which are expressed as tensor products of two quaternion 
algebras, where $K$ is a field of characteristic 2 . For instance let $A \cong Q_{1}^{\prime} \otimes Q_{2}^{\prime}$. Choose a Henselian valued field $F$ of characteristic 0 such that $\bar{F}=K$. If $Q_{i}$ is the inertial lift of $Q_{i}^{\prime}$ then $A=Q_{1} \otimes Q_{2}$ is the inertial lift of $A^{\prime}$. It also follows that the lift of the Albert form $\alpha_{A^{\prime}}$ is just $\alpha_{A}$. A similar analysis can be done for $B^{\prime}$. Assuming the characteristic 0 version of Jacobson's Theorem we find: $A^{\prime} \cong B^{\prime}$ over $K$ iff $A \cong B$ over $F$ iff $\alpha_{A}$ and $\alpha_{B}$ are similar over $F$ iff $\alpha_{A^{\prime}}$ and $\alpha_{B^{\prime}}$ are similar over $K$.

\section{REFERENCES}

[A1] A. A. Albert, Structure of algebras, Amer. Math. Soc. Colloq. Publ., vol. 24, Amer. Math. Soc., Providence, R.I., 1939.

[A2] __ Tensor products of quaternion algebras, Proc. Amer. Math. Soc. 35 (1972), 65-66.

[B] R. Baeza, Quadratic forms over semilocal rings, Lecture Notes in Math., vol. 655, SpringerVerlag, Berlin and New York, 1978.

[JW] B. Jacob and A. Wadsworth, Division algebras over Henselian fields, J. Algebra (to appear).

[J] N. Jacobson, Some applications of Jordan norms to involutorial simple associative algebras, Adv. in Math. 48 (1983), 149-165.

[K] M. Knebusch, Isometrien über semilokalen Ringen, Math. Z. 108 (1969) 255-268.

[L] T.-Y. Lam, The algebraic theory of quadratic forms, Benjamin, Reading, Mass., 1973.

[M1] P. Mammone, p-Algèbres et formes quadratiques, Doctoral Dissertation, Université de Mons, Mons, Belgium.

[M2] __ Similitude de formes quadratiques et corps de fonctions en caractéristique 2, Bull. Soc. Math. Bel. 39 (1987), 373-377.

[P] A. Pfister, Quadratische Formen in beliebigen Körpern, Invent. Math. 1 (1966), 116-132.

[R] M. L. Racine, A simple proof of a theorem of Albert, Proc. Amer. Math. Soc. 43 (1974), 487488.

[Sa] C. H. Sah, Symmetric bilinear forms and quadratic forms, J. Algebra 20 (1972), 144-160.

[S] W. Scharlau, Quadratic and Hermitian forms, Springer-Verlag, Berlin and New York, 1985.

[W1] A. Wadsworth, Similarity of quadratic forms and isomorphism of their function fields, Trans. Amer. Math. Soc. 208 (1975), 352-358.

[W2] _ _ Discriminants in characteristic two, Linear and Multilinear Alg. 17 (1985), 235-263.

Départment de Mathématique, Université de l'Etat á Mons, Avenue Maistriau, 15, 7000 Mons (BELGIQUE)

Department of Mathematics, Ohio State University, Columbus, Ohio 43210 